\title{
Bryophytes of the Čantoria National Nature Reserve (Moravskoslezské Beskydy Mts., Czech Republic)
}

Vítězslav Plášek

Bryophytes of the Čantoria National Nature Reserve (Moravskoslezské Beskydy Mts., Czech Republic). - Acta Mus. Siles. Sci. Natur., 64: 191-192, 2015.

\begin{abstract}
Bryological investigation in National Nature Reserve Čantoria was done in 2013. The total of 99 bryophytes (29 liverworts and 70 moss species) recorded and/or collected are listed in this contribution. The most important records included 2 liverworts (Liochlaena lanceolata and Nowellia curvifolia) and 4 mosses (Brachydontium trichodes, Campylostelium saxicola, Fissidens pusillus and Oxystegus tenuirostris).
\end{abstract}

Keywords: bryoflora, mosses, liverworts, Beskydy Mts, Northeastern Moravia, Silesia

National Nature Reserve Čantoria includes mainly primeval forest communities on the southern slope of the Velká Čantoryje Mt (Moravskoslezské Beskydy Mts). The protected area is situated NE of Nýdek village of along the Czech - Polish border. The reserve was declared in 1998 on an area of 39.45 ha at elevations of 720 - 958 metres a.s.l.

Bryofloristic survey of the area was carried out by author during spring and summer 2013. In total 99 taxa of bryophytes (29 liverworts, 70 mosses) were recorded there. All herbarium specimens are housed in OSTR.

\section{A list of recorded species}

\section{Liverworts}

Barbilophozia hatcheri

*Bazzania trilobata

Blepharostoma trichophyllum

Calypogeia azurea

Calypogeia integristipula

Cephalozia bicuspidata

Chiloscyphus coadunatus

Chiloscyphus profundus

*Conocephalum conicum

*Diplophyllum albicans

*Frullania dilatata

Lejeunea cavifolia

Lepidozia reptans

*Liochlaena lanceolata

*Lophozia ventricosa

\section{Mosses}

*Amblystegium serpens Atrichum undulatum Brachydontium trichodes Brachythecium populeum Brachythecium reflexum * Brachythecium rivulare Brachythecium rutabulum Brachythecium salebrosum Brachythecium velutinum Bryum moravicum *Campylostelium saxicola

\author{
Metzgeria furcata \\ * Nowellia curvifolia \\ Orthocaulis attenuatus \\ Pellia epiphylla \\ *Pellia neesiana \\ Plagiochila porelloides \\ *Porella platyphylla \\ *Ptilidium ciliare \\ Ptilidium pulcherrimum \\ * Radula complanata \\ * Scapania irrigua \\ Scapania nemorea \\ *Schistochilopsis incisa \\ Solenostoma gracillimum
}

\author{
Ceratodon purpureus \\ Ctenidium molluscum \\ Cynodontium polycarpon \\ Dicranella heteromalla \\ Dicranodontium denudatum \\ Dicranoweisia crispula \\ Dicranum montanum \\ Dicranum scoparium \\ Ditrichum heteromallum \\ Ditrichum lineare \\ *Dorcadion affine
}


*Dorcadion speciosum

Eurhynchium angustirete

Eurhynchium hians

Fissidens pusillus

Grimmia hartmanii

Herzogiella seligeri

*Heterocladium heteropterum

*Homalia trichomanoides

Hypnum cupressiforme

Isothecium alopecuroides

*Leucobryum glaucum

*Leucodon sciuroides

*Mnium hornum

Mnium stellare

*Orthotrichum anomalum

*Orthotrichum pallens

*Orthotrichum pumilum

Orthotrichum stramineum

Oxystegus tenuirostris

Paraleucobryum longifolium

Plagiomnium affine

*Plagiomnium undulatum

Plagiothecium curvifolium

Plagiothecium laetum
Plagiothecium nemorale

* Plagiothecium undulatum

*Platygyrium repens

Pleurozium schreberi

Pogonatum aloides

Pogonatum urnigerum

Pohlia nutans

Polytrichastrum formosum

*Polytrichum juniperinum

* Pseudoleskeella nervosa

Pterigynandrum filiforme

*Pylaisia polyantha

*Racomitrium aciculare

Rhizomnium punctatum

Rhytidiadelphus squarrosus

Sanionia uncinata

Schistidium apocarpum

*Sphagnum girgensohnii

*Syntrichia ruralis

Tetraphis pellucida

*Thuidium tamariscinum

Tortella tortuosa

*Ulota bruchi

*Ulota crispa

In the study area, totally 99 taxa of bryophytes (29 liverworts, 70 mosses) were recorded. Six taxa are included into some of categories of the Red list of bryophytes of the Czech Republic (Kučera, Váňa \& Hradílek 2012). Among the "Lower-risk mosses” (LR-nt) belongs Liochlaena lanceolata and Campylostelium saxicola. Category of "Least concern - attention list“ (LC-att) is represented by Nowellia curvifolia, Brachydontium trichodes, Fissidens pusillus and Oxystegus tenuirostris.

Despite considerable sampling efforts, an occurrence of 17 species recorded here in the past (cf. Reichardt 1858; Milde 1961; Podpěra 1928; Vězda 1960; Plášek 2000a, 2000b; Plášek \& Stebel 2002) were not recently confirmed. On the other hand, 38 species (13 liverworts, 25 mosses) were recorded here for the first time in 2013 - marked by “*” in a list of recorded species.

Acknowledgements: The contribution is a part of a research project of the IET, reg. no. CZ.1.05/2.1.00/03.0100, Project LO1208 of the National Feasibility Programme I of the Czech Republic.

\section{References}

Kučera J., Váňa J. \& Hradílek Z. (2012): Bryophyte flora of the Czech Republic: update of the checklist and Red List and a brief analysis. - Preslia 84: 813-850.

Milde J. (1961): Uebersicht über die schlesische Laubmoos- Flora.- Bot. Ztg., Mohl Schlechtedal, Liepzig, 19: 148.

Plášek V. (2000a): Ohrožené a vzácné druhy mechorostů Slezských Beskyd - I. - Acta Facultatis Rerum Naturalium Universitatis Ostraviensis 6-7: 7-15.

Plášek V. [ed.] (2000b): Seznam mechorostů zaznamenaných v průběhu bryolichenologických dnů ve Slezských Beskydech. - Bryonora 25:10-13.

Plášek V. \& Stebel A. (2002): Bryophytes of the Čantoryjský hřbet range /Czantoria range/ and its foothills (Western Carpathians - Czech Republic, Poland). - Časopis slezského zemského muzea 51: 1-87.

Podpěra J. (1928): Zajímavý mechový nález na Moravě. - Příroda 21, př́íloha 23, Praha.

Reichardt H.W. (1858): Gibt folgende Mitteilungen. -Verh. Zoo-Bot. Ges. Wien, Band VIII., pp.58-62.

Vězda A. (1960): Nová lokalita mechu Anacamptodon splachnoides (Froel.) Brid. v Československu. - Acta Musei Silesiae 9:91-94.

Author's address: Víťzslav Plášek, Dept. of Biology and Ecology, University of Ostrava, Chittussiho 10, CZ-710 00 Ostrava, Czech Republic. E-mail: vitezslav.plasek@osu.cz 